Published as: Vanclooster Ann \& De Maeyer Philippe (2012) "Combining indoor and outdoor navigation: the current approach of route planners". In: Gartner Georg \& Ortag, Felix (Eds.): "Advances in Location-Based Services" (Lecture Notes in Geoinformation and Cartography) - Springer-Verlag Berlin Heidelberg. 8th International Symposium on Location-Based Services (21-23 november 2011, Vienna), pp. 283-304.

\title{
Combining indoor and outdoor navigation: the current approach of route planners
}

Vanclooster Ann, De Maeyer Philippe

Department of Geography - Ghent University

Krijgslaan 281 (S8), 9000 Gent, Belgium

\{Ann.Vanclooster; Philippe.DeMaeyer\}@UGent.be

\begin{abstract}
This paper studies the use of indoor infrastructures for navigation in several currently available route planners. In the context of an increasing dependence on positioning and navigation tools, a shift has taken place from solely outdoor applications to the indoor environment. Although location based services and indoor positioning techniques may have gotten increasing attention from research and commercial point of view, ubiquitous indoor navigation systems are not yet available on the market. With people moving seamlessly from indoor to outdoor, systems that integrate navigation in both will be the next challenge in navigational research. This paper contributes to this integration of the notion of indoor and outdoor space by studying its impact on route planners. A review of various case studies in multiple route planners has been carried out which reveal different aspects and requirements for the indoor-outdoor connection in way finding. Currently, mostly data constraints prevent the optimal use of all navigation routes. Additional problems were discovered with address matching methodologies influencing the exit choice of buildings (leading in some cases to sub optimal routing). Recommendations are made for future
\end{abstract}


enhancements based on the product to market implications to come to a better integration of indoor with outdoor infrastructures.

Keywords. Navigation, Indoor, Outdoor, Route planner

\section{Introduction}

Over the last decade, navigational tools have become more and more prevalent as a resource for reliable route planning and way finding. Generally, navigation requires tracking and guidance by a technical localization infrastructure, support of multiple navigation contexts (navigable and nonnavigable space description based on user and environmental constraints) and an appropriate (for the application level) and accurate topographic representation of space (Nagel et al. 2010). For outdoor navigational systems, these requirements have been achieved over the years by the development of the global positioning system for tracking and guidance, a more efficient and abundant data collection using mobile mapping technology and improvements in modeling and data storage (e.g. GDF standard). However, this effort has been solely centered on pure outdoor car navigation systems.

Although pedestrian navigation systems hold similar demands for route planning, their interpretation and specification to the pedestrian context calls for a specific and individual adaptation. This is induced by differences in context, environment, mode of locomotion, scale level and technology (Walton \& Worboys 2009). For example, pedestrians walk more freely in the available space. Modeling this by using the available outdoor transport networks doesn't completely reflect this freedom (Bogdahn \& Coors 2009). Second, pedestrians have access to both indoor and outdoor environments requiring route guidance in both. This implies availability of both indoor and outdoor data, technological support in indoor environments and a communal space model. Third, the seamless movement from pedestrians from indoor to outdoor has to come to light again in the developed navigational models and route finding applications. Fourth, a more constrained environment makes route guidance more arduous due to a change in scale level and a more challenging landmark recognition. Current and future indoor and combined indoor-outdoor navigation systems should be able to implement these specific requirements.

Literature shows that over the last decade various researchers have begun developing systems based on situation awareness and smart environments using location based services (Gartner et al. 2007, Huang et al. 2009). A recent boost in technological advancements for tracking people in indoor environments has lead to increasing possibilities for the development of 
indoor navigational models. However, this research has focused solely on the technological aspects of indoor positioning and navigation (Mautz et al. 2010). From the multiple techniques available for indoor positioning, no standard has developed yet because none of them fulfill all positioning requirements. Alternatively, several researchers have developed a wide variety of indoor navigational models ranging from abstract space models (Becker et al. 2009) and 3D models (Coors 2003, Li \& He 2008) to pure network models (Jensen et al. 2009, Karas et al. 2006, Lee 2001, Lee 2004) and ontological models (Anagnostopoulos et al. 2005, Lyardet et al. 2008, Meijers et al. 2005). While these models might be useful in specific situations, a general framework for indoor navigation modeling has still to reach full maturity (Nagel et al. 2010). At issue is that all the previously mentioned attempts remain solely applicable to indoor situations. In order to fully accommodate navigation, a connection with outdoor applications has to be made.

Most current endeavors to combine indoor with outdoor navigation are focused on tracking techniques; in particular the transition of positioning tools from indoor to outdoor environments. The majority of these efforts originated from robotic research (Pfaff et al 2008) and navigation of the visually impaired persons (Ran et al. 2004, Scooter \& Helal 2005). The NAVIO project (Retscher \& Thienelt 2004) is one of the few attempts focused on pedestrian indoor and outdoor navigation. It aims at developing a route modeling ontology, which provides both outdoor and indoor routing instructions by identifying and formally defining the criteria, actions and reference objects used by pedestrians in their reasoning for navigation routes (Tsetsos et al. 2007). However, the project focuses solely on location fusion (i.e. the aggregation of location information from multiple sensing elements) and user interfaces, again making the approach too narrow. In the modeling field, the most notable work is of Slingsby and Raper (2007) who model a part of the built environment with its immediate surroundings. However, their model is quite complex and not suitable for navigational applications. It is also confined to describing small scale areas. The above research overview shows has up until now no fully integrative approach for combined indoor-outdoor navigation has yet been thoroughly developed.

Apart from the theoretical research efforts, some LBS applications have already been developed as practical pedestrian navigation applications. Makkamappa (www.makkamappa.com) is a smart phone based mapping system which can be used for GPS tracking after uploading maps and making it GPS linked. Photomap (http://ifgi.unimuenster.de/archives/photomap/Home.html) uses a technique of photographing public maps for pedestrian outdoor navigation. Both applications 
are focused on outdoor pedestrian routing using continuous GPS tracking. PinWi (Löchtefeld et al. 2010) is a LBS system for pedestrian indoor navigation which uses photos of an indoor YAH-map as navigation model and dead reckoning for positioning. As this may be a worthwhile approach, it is only locally applicable and not comprehensive enough for being a general indoor routing application. It is also less accurate and disregards problems of availability and indoor-outdoor integration. With above practical implementations having their merit, they still are mainly restricted to the application goal. Before developing more models for combined routing, an evaluation has to be made of the practical implementation issues with the integration of indoor and outdoor routing.

The key purpose of this paper is to evaluate the current use of indoor infrastructures for way finding in common route planners. This is done to make an evaluation of the next necessary steps and current problems in indoor and combined indoor-outdoor routing applications. Route planners are one of the first applications to acknowledge the data requirements for indoor and combined indoor-outdoor navigation since they do not require the technological advancements indispensable for full navigation applications. They focus mainly on the data and the presentation of the data in a certain data model used for traditional route calculations. Their implementation of indoor navigation requirements can serve as a base for practically improving current indoor and combined indoor-outdoor routing endeavors and for bringing theory closer to practice.

In this paper, first a review has been carried out of various case studies in multiple route planners, which reveals different aspects and requirements for the appropriate indoor-outdoor connection in way finding. The case studies each examine a current problem in the indoor-outdoor connection by comparing the results of the most commonly used route planners. Secondly, results of this review and their mutual comparison are employed in the discussion to reflect on recommendations for a better future use and integration of indoor infrastructures in route planning applications.

\section{Route planner review}

The objective of this review is to grasp the current state of the art on the integration of indoor infrastructures for navigation in common route planners. Without a proper connection of indoor with outdoor environments for navigation, route planners may calculate non accurate and sub-optimal routes. In this review, indoor infrastructures are considered buildings with multiple entrances above and underground, underground walkways, underground shopping centers and underground transportation systems. 
Since the indoor built environment can only be accessed by pedestrians, only pedestrian navigation is taken into account with a possible connection to public transport options. The used route planners are common for way finding within the geographical area of the query. For queries in Belgium, the following route planners are used:

- Bing: www.bing.com/maps

- Google Maps: www.googlemaps.com

- Mappy: www.mappy.com

- Via Michelin: www.viamichelin.com

- RouteNet: www.routenet.com

- OpenRouteService: http://openrouteservice.org

Queries in Korea are performed with the use of Google Maps and Naver (maps.naver.com). In the different case studies, multiple aspects of the indoor-outdoor connection in routing will be investigated using various route planners. A comparison of the quality of the current route planners is assessed recording their approach of handling data.

\subsection{Indoor data availability}

Following examples all make use of an internal network structure. However, usage is not always straightforward or optimal.

\subsubsection{Indoor infrastructure as part of the shortest path}

To test whether a route planner utilizes the indoor network structure in the shortest path calculations, a first query has been executed to navigate from Cantersteen to Ravensteinstreet in Brussels (Belgium). The optimal pedestrian and shortest path route uses the Ravenstein gallery with aboveground entrances in both streets.

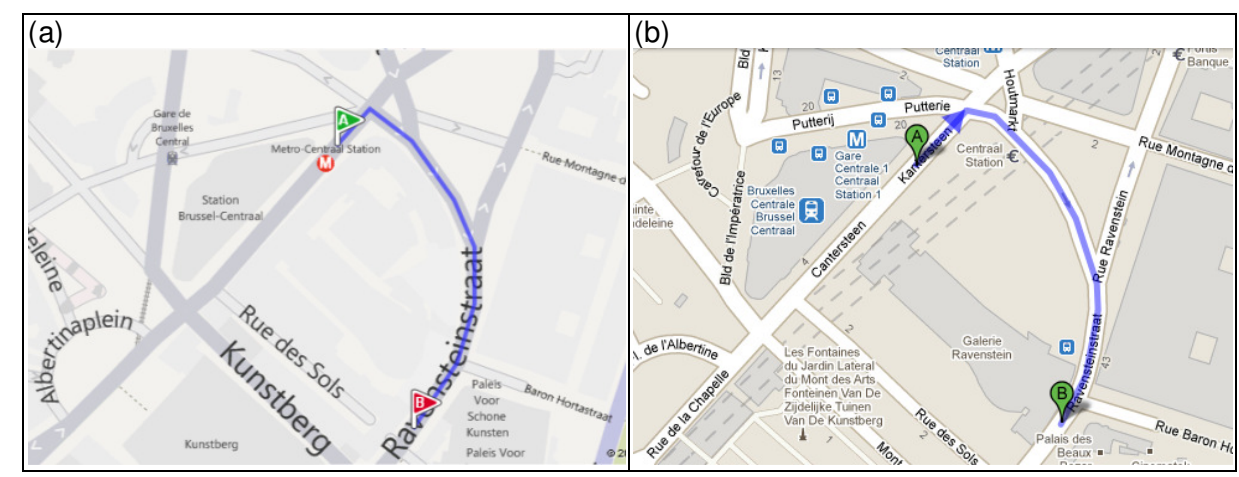




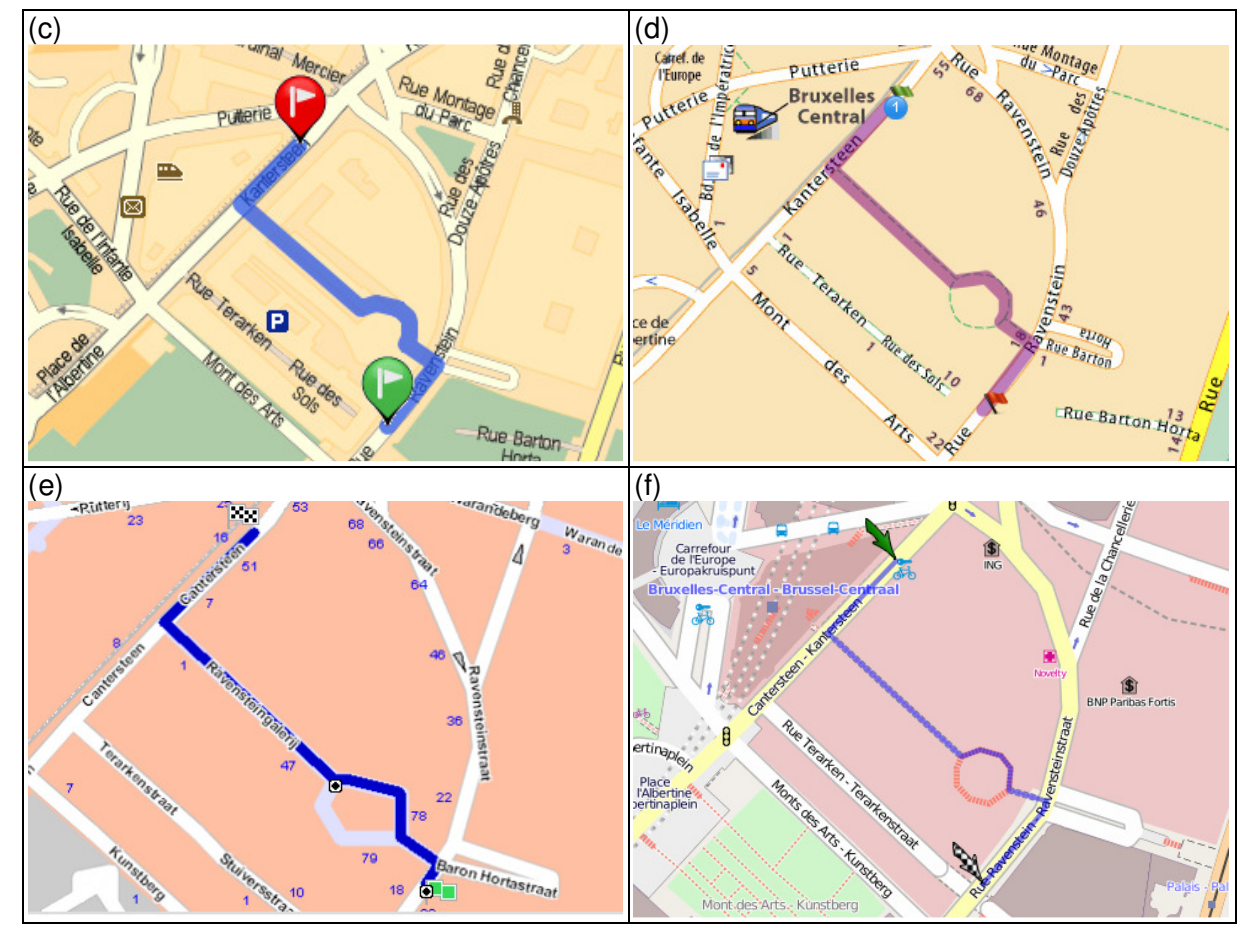

Figure 1. Navigation from Cantersteen to Ravensteinstraat (Brussels, Belgium) using route planner (a) Bing ,(b) Google Maps, (c) Mappy, (d) Via Michelin, (e) RouteNet and (f) OpenRouteService.

Differences over the multiple route planners can be detected. Both Bing and Google Maps don't make use of the gallery, while Mappy, Via Michelin, RouteNet and OpenRouteService on the other hand do. It can be noted that Bing doesn't even recognize the gallery as part of the spatial dataset. In Google Maps the gallery is mapped with a text label, but is not part of the vector data available for routing. The other route planners map the optimal and shortest pedestrian route between departure and destination point. This query shows that in some cases both the indoor network structure and the aboveground entrances are mapped and used in the calculation of the shortest path.

A second example studies the use of an underground structure as part of a shortest path calculation in Myondong underground shopping centre (Seoul, Korea). The route planner was asked to perform a route calculation from the Lotte Department Store in Myongdong to the Ibis Hotel across the 
street. This street is not directly crossable by pedestrians due to heavy traffic. Instead, across the hotel entrance is an underground passage way and shopping centre which leads to the other side of the road.

With this query the usability of $3 \mathrm{D}$ underground structures in route planners (both the location of entrance points and network usage) is tested. For this query, local data for the city centre of Seoul was only available through Google Maps and Naver (a Korean route planner), while other route planners lacked detailed street network data.

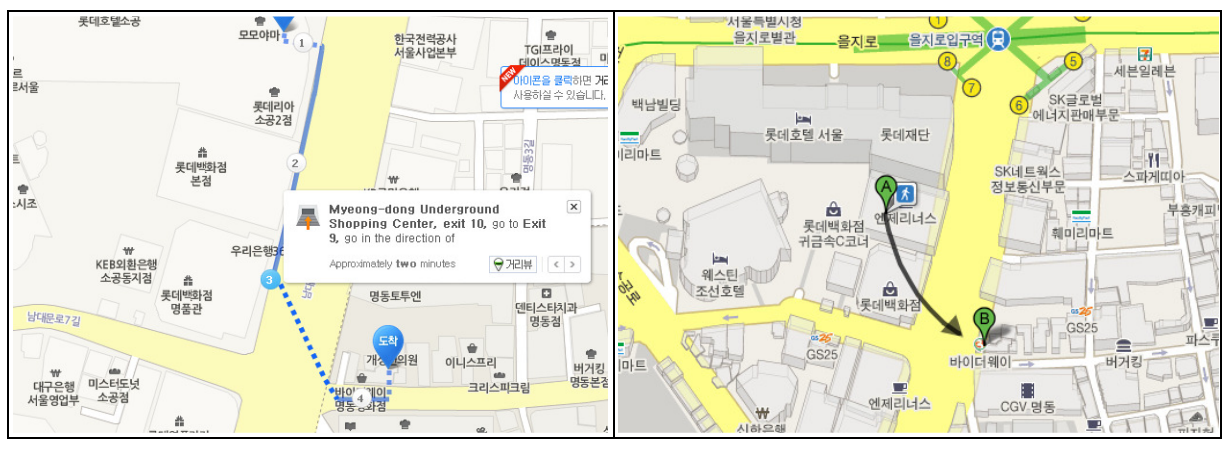

Figure 2. Navigation from Myongdong Lotte Department Store to Ibis Hotel (Seoul) using Naver (left) and Google Maps (right).

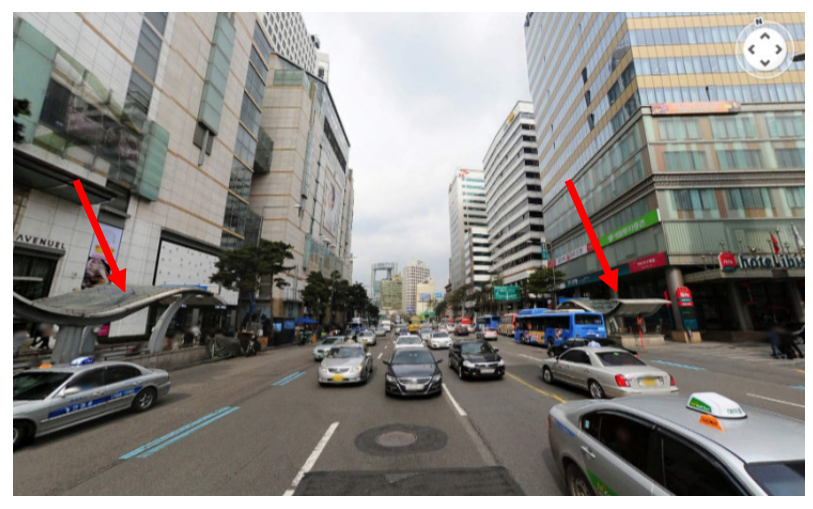

Figure 3. Street view of road in Myongdong (Source: Naver). The red arrows show the entrances of the underground passage way.

This example shows that there is a huge difference in navigational instructions for both route planners. While Google Maps doesn't provide routing information for pedestrians in Seoul, Naver on the other hand has very de- 
tailed information of the available pedestrian roads. It recognizes the underground passage way with the corresponding entrance points and exit numbers. Consequently, the navigation instruction is described incorporating all possible details.

\subsubsection{Availability of entrance information}

Apart from checking the use of internal network structures, it is also interesting to verify the data completeness of the route planners for navigation. Interior data can be considered complete if it can solve all queries, has the appropriate interior network edges, semantic information and ability to connect the indoor with the outdoor networks via the entrance/exit points of buildings. As is shown in the first example of section 2.1.1, Mappy, Via Michelin, RouteNet and OpenRouteService use all aboveground entrances in the calculation of the shortest path. However, the gallery also has one underground connection with the main railway station in Brussels. The following query tests the use of this underground entrance with a query from the railway station to the Ravenstein gallery. The query is executed in all six available route planners.

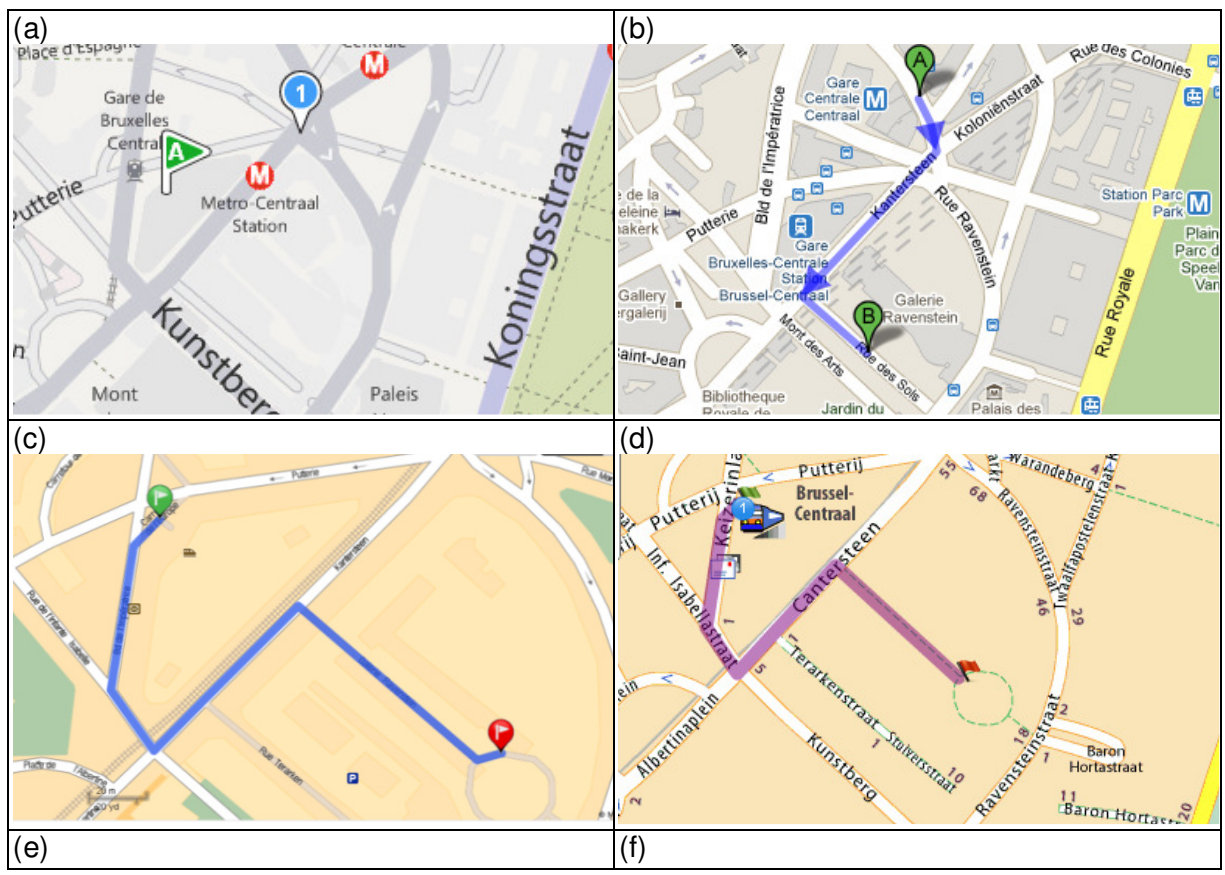




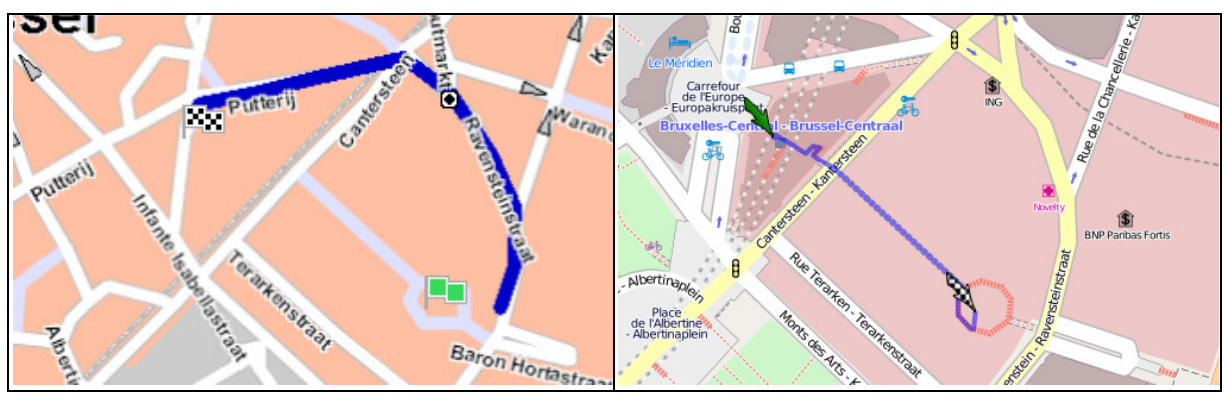

Figure 4. Navigation from Brussels Central Station to Ravensteingallerij using route planner (a) Bing ,(b) Google Maps, (c) Mappy, (d) Via Michelin, (e) RouteNet and (f) OpenRouteService.

It can be concluded that only OpenRouteService provides all the entrances to the indoor gallery, even the underground passage way. The spatial data sets of the other route planners are incomplete resulting in sub-optimal routing instructions. It has to be pointed out that the address matching (discussed in Section 2.2) influences the ability to calculate the routes. For the query in OpenRouteService, the start position has been manually pointed out, since this route planner does not incorporate appropriate address matching. In the Bing route planner, accurate data is lacking of the building itself (attribute is not found in the dataset), making it impossible to even calculate a route. Google Maps has the attribute information but the address is not linked to the network. Instead, the endpoint is linked to the closest available network data with respect to the central point of the gallery. Also, Google Maps links the attribute information for the Central Station to a different geographical location compared to the other route planners. Mappy and Via Michelin, on the other hand, both have network data inside the building complex. However, the underground passage way from the station to the gallery is not digitized. RouteNet maps the location of the gallery on the same postion. However, despite having the internal network structure, the calculated route leads to the back entrance which is the closest to the mapped location (i.e. the location of the address).

\subsection{Address matching}

In the following examples the query requires appropriate linking between the users input and geographical coordinates.

\subsubsection{Address matching within indoor infrastructures}

As shown in section 2.1.1, in some cases indoor network data is available. However, the availability of an indoor network is no guarantee for appropriate linking of indoor features with indoor address localization. In the 
following example this is tested through navigating within a certain indoor infrastructure which requires indoor addresses linked to the network structure. Note that we don't take into account the indoor tracking methods necessary for an indoor positioning system and solely focus on the navigational instructions of route planners. This case study is again carried out in the Ravenstein gallery in Brussels. As was concluded from the example above, only Mappy, Via Michelin, RouteNet and OpenRouteService were able to visualize and use the indoor network in its route calculations. Therefore only those are used in the current example.

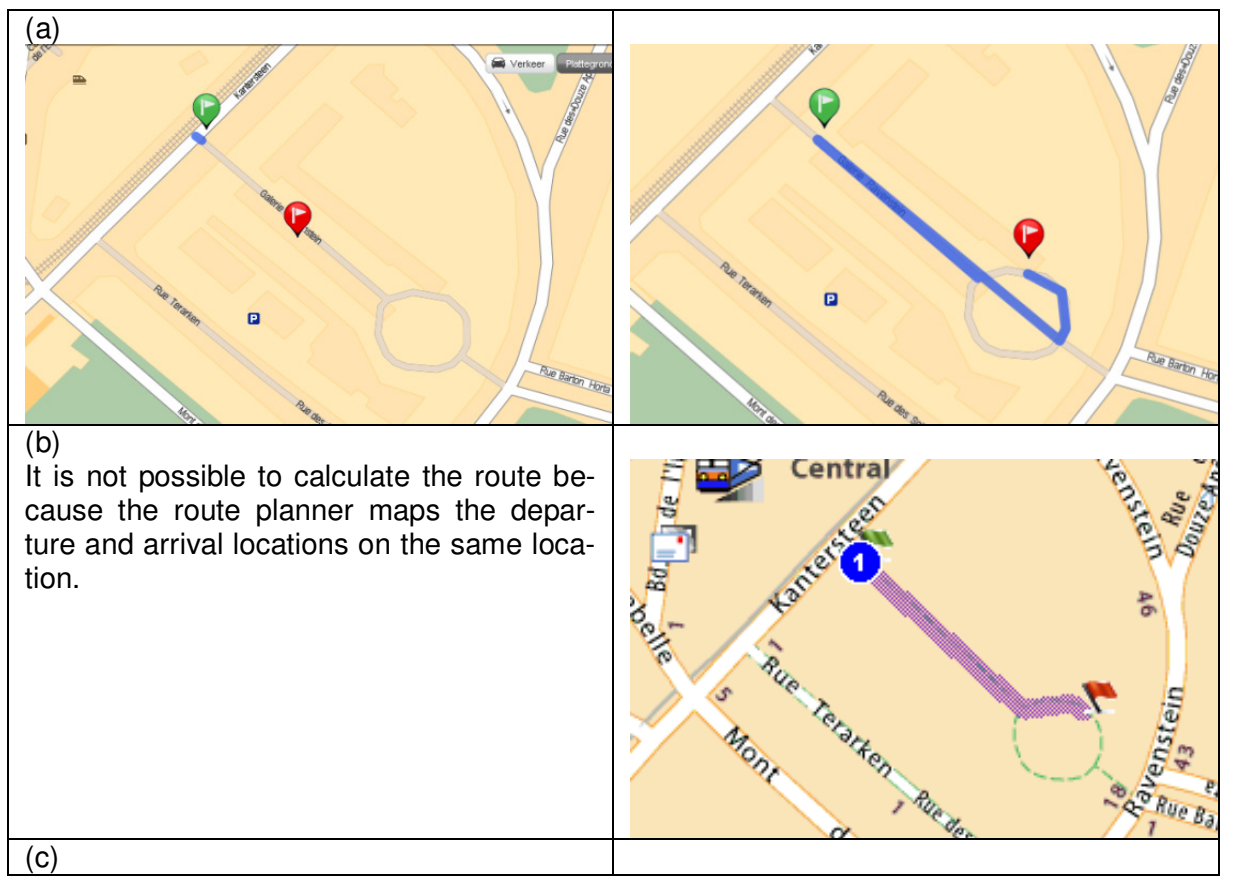




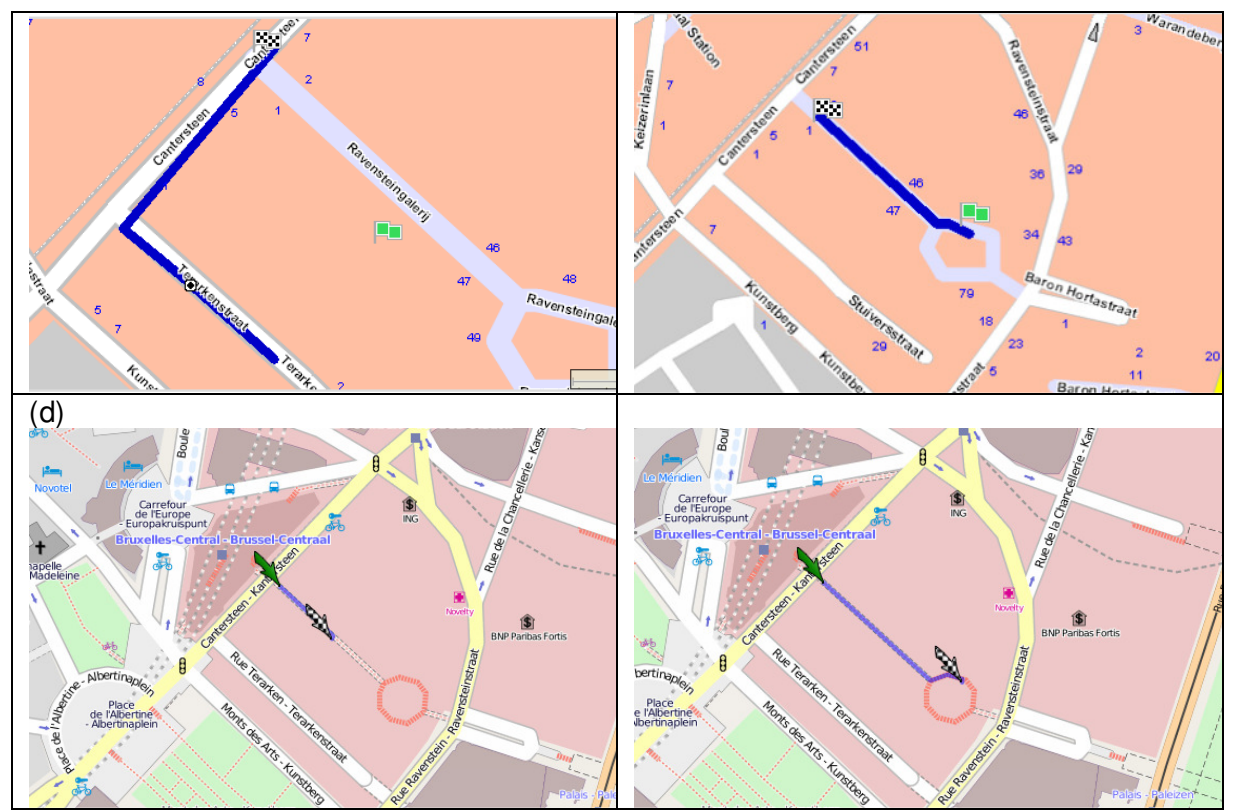

Figure 5. Navigation from Ravenstein gallery 2 to Ravenstein gallery 27 (left column) and from Ravenstein gallery 12 to Ravenstein gallery 60 (right column) using (a) Mappy, (b) Via Michelin, (c) RouteNet and (d) OpenRouteService.

These similar queries lead to different results over the various route planners. With the navigation instructions in the left column, both destination and departure points are situated on the same network edge which requires a linear interpolation technique for appropriate address matching. OpenRouteService completely lacks a link between addresses and spatial location. Even for outdoor environments, specific addresses in the same street are linked to one point on the network. For this query, the position of start and destination were added manually. The calculation of the shortest route makes use of the internal network. OpenRouteservice can as a consequence not be used for accurate address matching.

As can be seen, only Mappy and RouteNet are able to visualize the correct end points. However, none of them are able to actually calculate the shortest route between them. They both use a different mapping method to project the end points to the correct position on the network. Mappy maps the correct internal location, but cannot connect them through the indoor network. RouteNet searches for the closest available network edge to map the address and connects them using the outdoor network.

The second query also requires internal navigation in the same gallery, but the end point is located on a different part of the internal network. As can 
be seen from Figure 5 (right column), in this case all route planners are able to perform a correct address matching with a proper connection to the interior network. Via Michelin and RouteNet calculate the shortest path between both points, while Mappy uses a part of the network twice in its calculations resulting in a sub-optimal navigation solution.

\subsubsection{Address matching influences the exit choice}

Another aspect of the challenges involved with the indoor-outdoor connection is the way in which exit points and address matching methods are related to each other. The next two case studies test whether route planners make use of different exit points of indoor infrastructures when calculating routes to different locations and in what way the exit choice influences the final route calculation.

This first example uses the main station in Ghent (Belgium) as starting point for two queries. The first query (left column in Figure 6) asks the route to the centre of town, north of the station. The second query (right column in Figure 6) requires the route to the hospital in the south of the city. The station has two main entrances, one at the front (north side) and one at the back (south side) of the station.

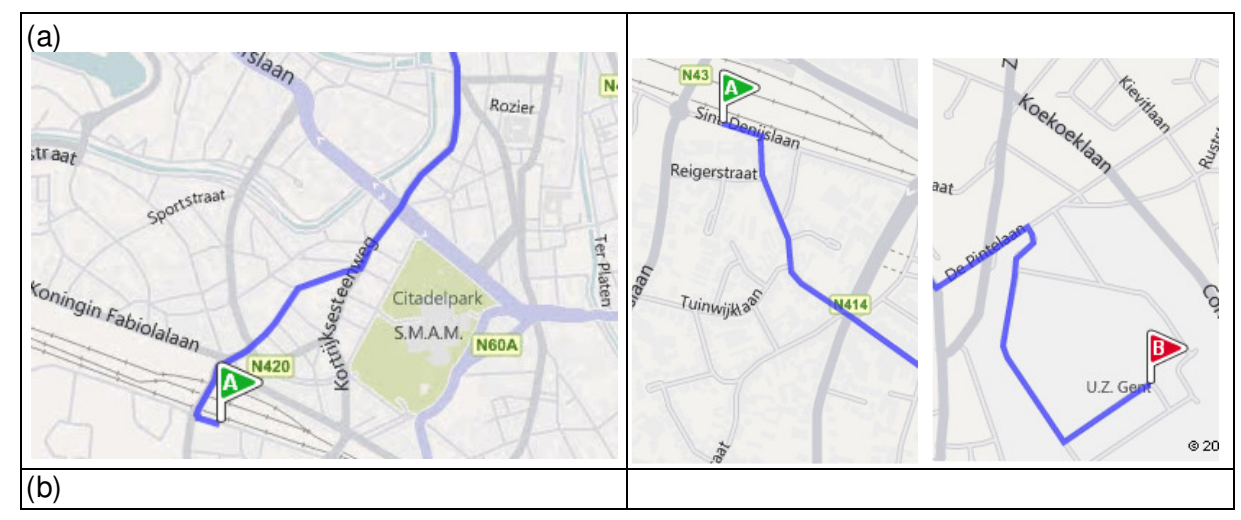




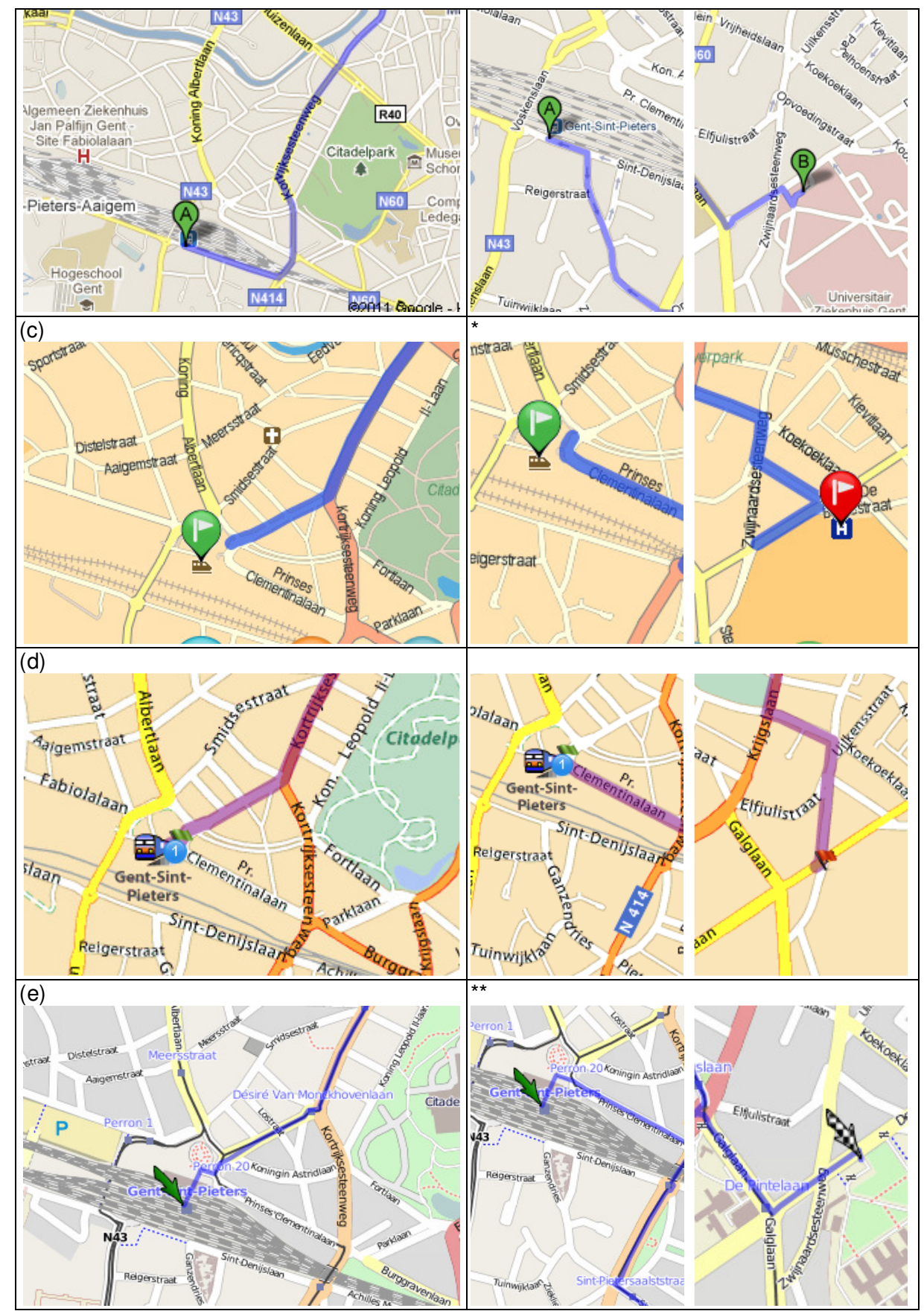


Figure 6. Navigation from railway station Gent-Sint-Pieters to Korenmarkt (left column) and University Hospital (right column) using multiple route planners (a) Bing, (b) Google Maps, (c) Mappy, (d) Via Michelin and (e) OpenRouteService.

*Via Michelin didn't recognize the name 'UZ Gent' or 'Universitair Ziekenhuis Gent'. Instead the address given by the website of the hospital (De Pintelaan 185) is used as end point of the query.

**OpenRouteService doesn't incorporate appropriate address matching capabilities. The start and end points of the queries are added manually.

From above example it can be concluded that all five route planners only use one entrance/address point for route planning, no matter what the destination of the query is. Both Bing and Google Maps have the station located at the back entrance, making the route to the city centre not optimal. Interestingly enough, in this case they even use different solutions to get to the north side of the station, due to different routing algorithms used in the calculation. For the second query, the departure points with respect to the geographical location of the station remain the same over all route planners. When looking at the destination, the different route planners use multiple locations depending on the availability of the spatial data.

A second case study takes place in the Waasland shopping centre in SintNiklaas (Belgium). It is not so much focused on indoor networks, however the results can have major importance for future indoor-outdoor connections. The query inquires about driving directions to the shopping centre. The shopping centre has multiple entrances and parking spaces which makes driving rather complex. One of the problems here is the question of where to park your car when you want to go to a certain shop. A certain optimization can take place which requires the connection of the several entrances, the internal building layout and the immediate outdoor environment.

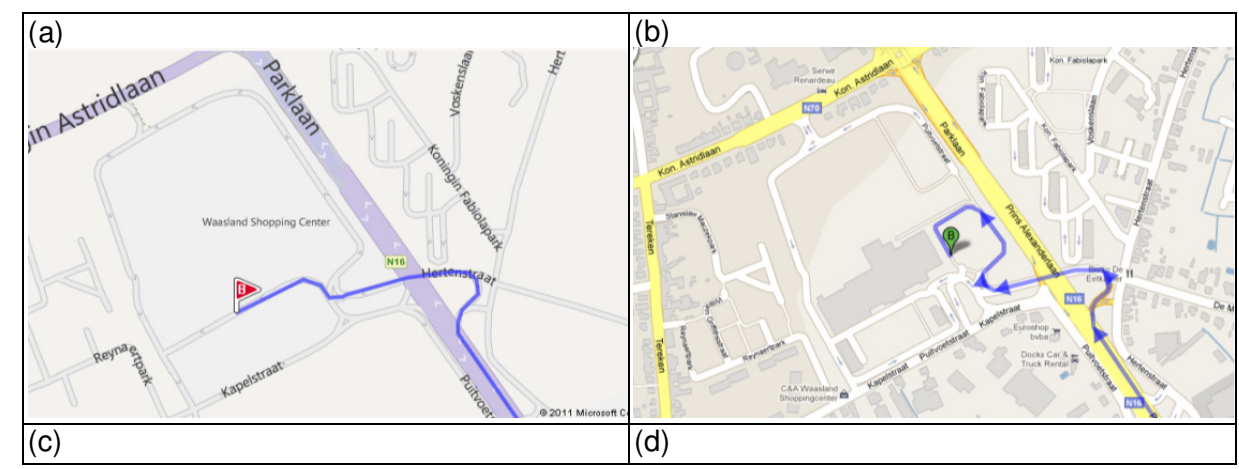




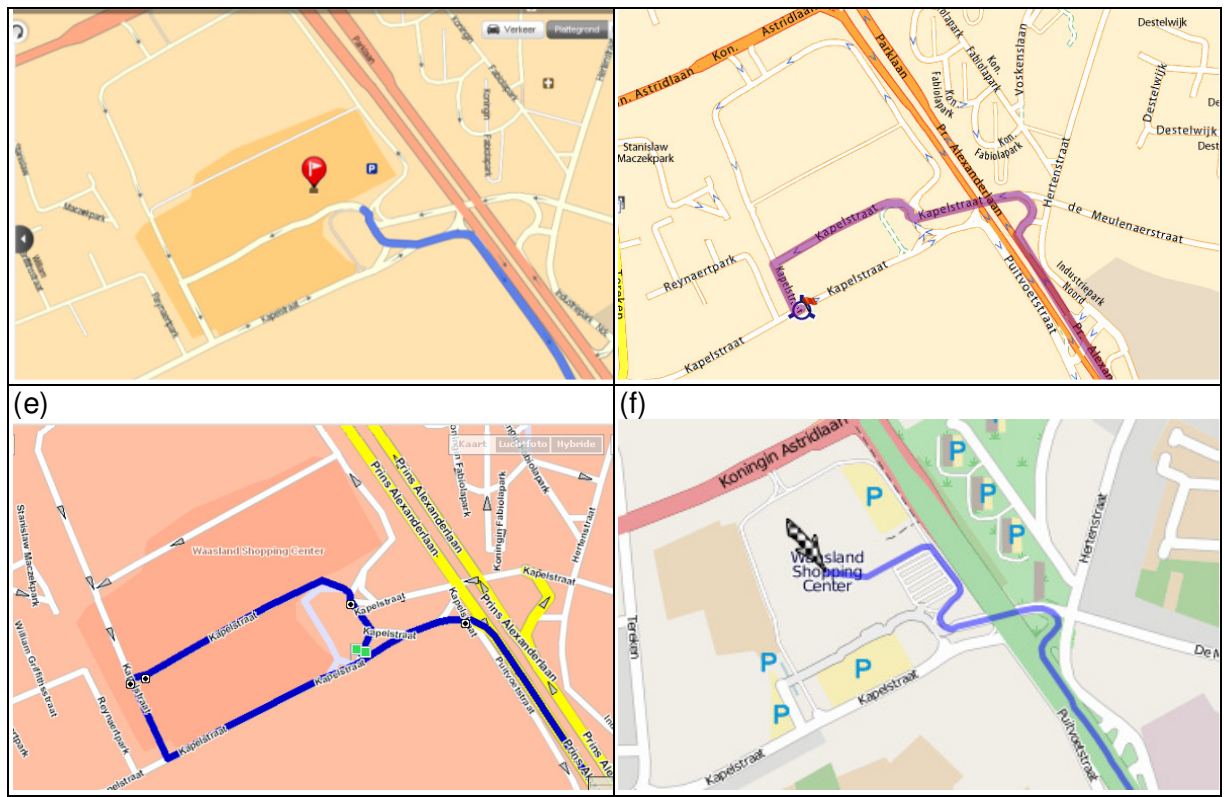

Figure 7. Driving instructions to Waasland Shopping Centre using (a) Bing, (b) Google Maps, (c) Mappy, (d) Via Michelin, (e) RouteNet and (f) OpenRouteService.

It can be seen that the geographic location of the endpoint differs over the various route planners. The digitalization of the outdoor parking area varies from quite rough (Bing) to very detailed (Google Maps). However, none of the route planners make use of entrance point information, making a future indoor-outdoor connection at the moment rather difficult. The algorithm for linking the address information with the spatial network information differs for every application, but is of major importance for results of the route calculations.

\subsection{Multimodal routing application}

One of the applications where the indoor-outdoor connection in navigation is really important is when changing mode of locomotion and this mostly related to the public transportation system. In the following case study a multimodal path using public transportion is calculated from Donuidong 30 to the University of Seoul (Seoul, Korea). The calculated route involves changes from pedestrian movement to subway and bus. The first part of the route consists of the movement from the address to the subway entrance. Both route planners make use of the same subway line. 


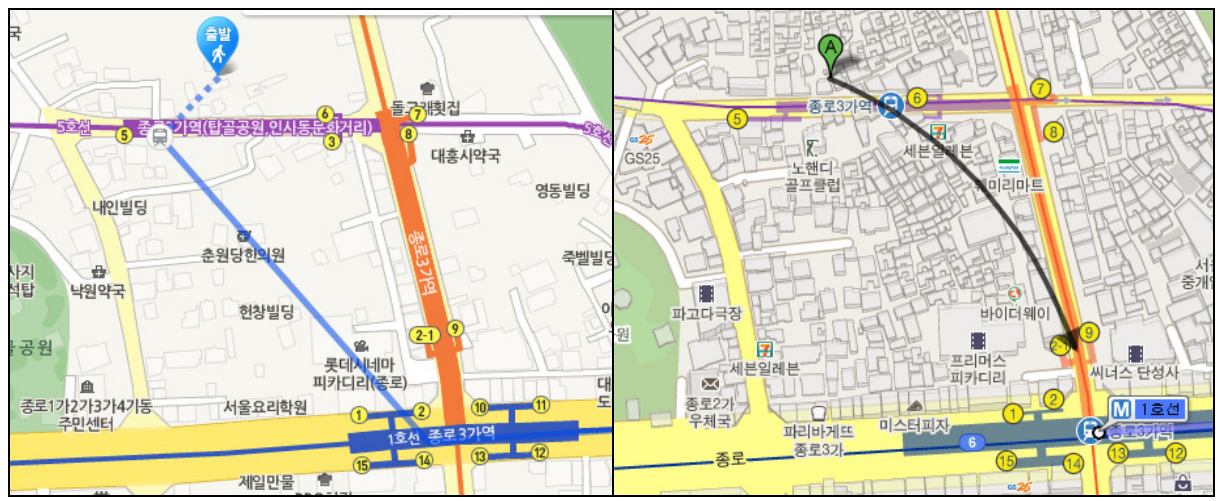

Figure 8. Navigation from Donuidong 30, Seoul to University of Seoul in Naver (left) and Google Maps (right). Zoom of part 1 from Donuidong 30 to Jongno 3-ga subway line 1.

With above routing navigation, we can make the following conclusion:

Google Maps doesn't support detailed and accurate navigational instructions, only the information to go to subway line 1 with stop Jongno 3-ga. Naver on the other hand is more detailed and connects the walkway from the given address to the entrance of subway line 5 (Jongno 3-ga). The route is continued using the underground subway infrastructure until line 1 is reached. However, details from within this underground infrastructure are not provided.

With the above example, it is shown that Naver knows the available underground structures and entrances. However, the entrance choice is solely based on the shortest route aboveground. In reality, when entering the subway of Jongno 3-ga at entrance 4, the route requires descending over multiple floors and is much longer and more exhausting to walk than walking directly towards entrance 6 . As is shown here, knowledge of $3 \mathrm{D}$ underground obstacles and structures does affect the optimal route choice but is currently not taken into account.

\section{Discussion}

In the following paragraphs, we will first discuss some more general conclusions with regard to the previously described case studies. We follow the same structure of the examples given. Subsequently, some of the implications and difficulties for immediate development of indoor routing are being discussed. 


\subsection{Problems with current indoor navigation applications}

From the above case studies, several conclusions can be drawn.

First, with regard to the data availability and completeness of the data we can conclude that most route planners do not incorporate indoor infrastructures in route calculations. This is most likely given by a lack of available indoor data (e.g. Bing in Section 2.1.1). Reasons for this are likely related to the fact that indoor data gathering has only just begun over the last few years. Also, the geographical area of the query could account for the unavailability of data in some areas, since companies developing route planners will put most effort into areas with the highest commercial value (e.g. European route planners have no detailed data available from the city centre of Seoul). Among route planners which do have some indoor data available, there is a dramatic difference in their level of detail. Data ranges from very rough (e.g. Google Maps in Section 2.1.1) to quite detailed (Naver in Section 2.1.1 and Section 2.3 and Mappy and Via Michelin in Section 2.1.1). When this indoor data is available, the disparate route planners mostly use it integrated with their outdoor networks in the shortest path calculations (Section 2.1.1).

The data problem is more pronounced with regard to underground structures. Usually both the entrance points and the underground network are not available (Section 2.1.2). Even with the most accurate information available, there are issues in calculating the optimal routes. Although the entrance location and attributes are used as connectors between outdoor and indoor network data, the actual underground network structure is not mapped or known. This results in a lack of knowledge about the $3 \mathrm{D}$ infrastructure which can have a detrimental effect on navigation instructions (no indication of how to move in the underground area requires the user to rely on the available exit signs or other information) and calculation of shortest path (the result is mostly not the shortest path because of the movement in 3 dimensions with entrance choice based on the shortest aboveground path). In that case, the route planner uses the knowledge of the various entrances of an underground system and the time needed to move from one to another to calculate the shortest routes.

Secondly, the discussion from Section 2.2 implies that address matching is a problem for both outdoor as well as for indoor navigation. Outdoor address matching links the address to a single entrance/exit point, no matter what the destination of the query is. Not differentiating between the start point of the query with respect to the destination leads to inaccurate routing. Indoor address matching is done through linear interpolation of the indoor network structure (if available). When no indoor infrastructure is available, addresses are matched through projecting the central point on 
the closest outdoor network edge (see Section 2.2.1 and Section 2.1.2). The accuracy of the storage and location of the addresses is thus of major importance for routing in general and can highly influence optimal routing calculations.

Third, the connection of indoor and outdoor networks is mostly guaranteed when the travel mode remains the same and the entrance data is available (see Section 2.1.1 and Section 2.3). However, changing of mode of locomotion influences the route calculation making the calculations more complex (see Section 2.2.2 shopping centre example). This depends on both the data quality of the indoor-outdoor connection as well as the general accuracy of the outdoor network. This will be an issue for the future expansion of indoor-outdoor navigation applications with optimizations of route calculations.

\subsection{Indoor navigation: product-to-market implications}

\subsubsection{Data acquisition, standards and accuracy}

Data is the main ingredient for navigation and route planning. Within the area of outdoor navigation applications, a wide variety of data sources is already available from a mix of local and global data providers. The main spatial data providers are Navteq, TeleAtlas and Google. Historically, Holland-based TeleAtlas and American Navteq were interwovenly used in many navigation applications. However, purchases lately of the main data providers by commercially independent navigation producers (Navteq by Nokia and TeleAtlas by TomTom) resulted in individual vouching for your own data set. As a result, Google (who had just signed a deal for using TeleAtlas data) switched to individually conducted data gathering for their US dataset. Additional reasons for this move, were said to be the lack of accuracy and coverage in the United States from the TeleAtlas data (http://blumenthals.com/blog/2009/10/12/google-replaces-tele-atlas-

data-in-us-with-google-data/). Google increased with this step its intention as one of the main contenders for spatial data information. From these data providers, no comprehensive efforts have currently been made to expand their spatial data set with ubiquitous indoor data.

As seen in the examples above, data is also crucial in the incorporation of indoor infrastructures in analysis and route calculation. The feasibility of indoor data acquisition is in this regard challenged and unseen. Nowadays, the available spatial datasets are mainly being updated and created using aerial images an mobile mapping vans. These methods are however not suitable for indoor mapping. Technically, a consensus is still lacking on a universal indoor tracking method as solution for the unavailability of GPS 
signals in buildings. One of the results is that the currently used user input from GPS tracks for updating and editing OpenStreetMap data cannot be applied here unless a ubiquitous indoor tracking system has been developed. Other options for indoor data gathering include photo modeling and laser scanning of individual buildings (Biber et al. 2004); but this is work intensive, expensive and not a comprehensive way of solving the data problem. Currently, many indoor data already exists in the form of for example YAH maps, CAD plans, CityGML or IFC models. These data represent the topographic building structure developed from certain application fields (e.g. structural building development, orientation purpose, evacuation maps). The problem with these indoor data sources is the huge diversity in data structure, completeness, availability, data coverage and level of detail. The area and institutional rules of the country also influence the specificity of the data source. As long as no generally accepted indoor standard is developed or a method to incorporate every possible indoor data source, comprehensive indoor data inclusion will remain challenged (Nagel et al 2010).

In either way, from these data sources correct networks have to be deducted. Since there is still no consensus on an appropriate and mathematically sound relation between data source and network creation for indoor environments, this is an additional problem needed to be solved before real indoor navigation can happen (Nagel et al. 2010). From the OGC and research environment attempts are currently made to develop a general framework and data standard (similar to GDF) for indoor navigation (Nagel et al. 2010). This is a promising step towards creating a background data model which can be used independently of the data input source.

\subsubsection{Indoor geocoding challenges}

A second major challenge in indoor navigation and route planning, is the geocoding of the users input to a geographical location or spatial unit. The term geocoding refers to assigning a geographic code based on a certain input information. Mostly geocoding is synonymous with address matching, arising from the prevalent use of transforming postal addresses into geographic coordinates (Goldberg et al. 2007). However, the input source can contain any other type of locational data (e.g. named buildings). Apart from the input, the fundamental components of the geocoding methodology include the processing algorithm, the reference dataset and the requested output (Goldberg et al. 2007). The challenges with the processing algorithm include identification of the separate parts of the input consistent with the reference data set (i.e. standardization and normalization process), matching of the best candidate with reference to the input data and determination of the appropriate geocode for output (Goldberg et al. 2007). The reference dataset consists of the data with which the input data will have to be 
matched. The output can be any geographically referenced object matching with the input data (Goldberg et al. 2007).

Goldberg et al. (2007) mention frequently induced errors in the outdoor geocoding methodology. With the most commonly used linear interpolation techniques, several assumptions are already made that affect the resulting geocoding accuracy (e.g addresses are assumed to all exists with equal parcel width). This methodology is also only restricted to outdoor address location finding, mostly on street level. However, other methodologies (e.g. area based or hybrid address matching) have similar problems and disadvantages. The reliance of 2D GIS data sources precludes the ability for highly precise geocoding of $3 \mathrm{D}$ structures with multiple addresses (Goldberg et al. 2007).

Indoor geocoding is susceptible for even more difficulties. First and foremost, the existing semi-uniformity in outdoor addressing is completely non-existing indoors due to country-related differences and a less rule based structure. For example, a 3D address consist of a 2D building address and a $3 \mathrm{D}$ subunit address, describing the location of a building's interior room (Lee, 2009). Lee (2009) suggests a $3 \mathrm{D}$ address geocoding methodology. It is based on a two-step process with first determination of the building within the geographical area (following the outdoor geocoding methodologies), followed by a street-like linear interpolation technique applied on an internal network of the building. This approach disregards the problems of discontinuous room numbering, for which transition tables can be a solution. Secondly, a reference dataset for indoor environments is not available. Outdoor geocoding methods mostly use existing street network data set (e.g. TIGER) with the range of house numbers linked to the street intersection or spatial street feature in the database. As long as no standard for indoor data exist, reference datasets will not be available for address matching.

\subsubsection{General feasibility issues}

Concluding, we are still far apart from incorporating indoor environments in routing applications. Challenges remain in data availability, storage, network completeness, linkage to the outdoor networks and geocoding. Technical innovations, research and creativity in the routing with less data might improve the feasibility for success in the next years. It is shown that the availability and quality of outdoor and indoor data and their connection is of high importance for the resulting route calculations. It appears that it is not feasible to gather and maintain all indoor data accurately from all buildings in the next years, since this would require a huge amount of data collection and maintenance. However, such a complete data gathering is 
not always necessary. Even small enhancements in indoor data can have a huge influence on routing (e.g. pointing out all connection points between indoor and outdoor environments, even without the actual indoor network would make the address matching more accurate and would also provide possibilities to have more optimal routes as for example shown in Section 2.3). More accurate information will of course result in optimal route calculations.

With all the above mentioned challenges, it is not possible to do a complete data acquisition for a combined indoor-outdoor navigation. We should seek to focus on large infrastructures and transportation networks with more specific navigational directions. The benefit of accommodating navigation in those infrastructures is bigger since a lot of people daily use and rely on those. These structures are also quite often fixed and stable over long periods of time, making the indoor data gathering and maintenance also more feasible. As is shown in the examples, the 3 dimensional network aspect is here of major importance to enhance routing for everyone.

An important role in data acquisition and address matching will be for the public. Over the last year, an increase has been seen in the public participation for outdoor data following the success of the data acquisition in OpenStreetMap (i.e. Wikipedia style updating and editing of data). This was noticed and built upon by other internet based applications and could also be a solution for indoor routing applications. Already at this moment users can change addresses and location of addresses for outdoor routing. Once the technology is ready for continuous indoor tracking and more user input is allowed, this could open up the indoor world too.

\section{Conclusion}

With this comparison of how current route planners use indoor infrastructures in the calculation of pedestrian routes, several active problems with this indoor-outdoor connection are identified. The most stringent limitation of current route planners in this realm is the availability of accurate data of indoor infrastructures. This data should consist of network information, additional semantic enrichments and all entrance points. As can be seen from the examples above, nonexistent or inaccurate information can lead to sub optimal routing, and even to a lack of routing in many cases. However, when the appropriate data is available, very precise routing information is proven to be calculated. It is pointed out that even small data additions, such as entrance and exit points of major infrastructure projects, can have a huge influence for pedestrian routing. Secondly, outdoor address matching techniques cannot directly be applied to indoor datasets. Immediate in- 
door-outdoor connection for navigation applications still have a long way to go. This research fits in with the ongoing awareness of indoor and outdoor navigation and more specifically it gives an overview of the data requirements for navigational applications. Future applications will more often focus on this indoor-outdoor connection, not only in navigation but also for wider analyses and applications.

\section{Acknowledgements}

Financial support from the Flanders Research Foundation (FWOVlaanderen) is gratefully acknowledged.

\section{References}

Anagnostopoulos C, Tsetsos V, Kikiras P, Hadjiefthymiades SP (2005) OntoNav: A Semantic Indoor Navigation System. Department of Informatics and Telecommunications, University of Athens. http://sunsite.informatik.rwth-aachen.de/Publications/CEUR-WS/Vol16.5/paper14.pdf. Accessed 13 May 2011

Becker T, Nagel C, Kolbe TH (2009) Supporting contexts for indoor navigation using a multilayered space model. Proceedings of the 2009 Tenth International Conference on Mobile Data Management: Systems, Services and Middleware: 680-685

Biber, P., Andreasson, H., Duckett, T., \& Schilling, A. (2004, 28 Sept.-2 Oct. 2004). 3D modeling of indoor environments by a mobile robot with a laser scanner and panoramic camera. Proceedings of the 2004 IEEE/RSJ International Conference on Intelligent Robots and Systems (IROS 2004).

Bogdahn, J., \& Coors, V. (2009). Using 3D Urban Models for Pedestrian Navigation Support. Paper presented at the GeoWeb 2009 Academic Track - Cityscapes, Vancouver, BC, Canada.

Coors V (2003) 3D-GIS in Networking Environments. Computers, Environment and Urban Systems 27(4): 345-357

Gartner G, Cartwright W, Peterson MP (2007) Location Based Services and Telecartography. Springer-Verlag, Berlin Heidelberg

Goldberg, D. W., Wilson, J. P., \& Knoblock, C. A. (2007). From text to geographic coordinates: the current state of geocoding. Journal of the Urban and Regional Information Systems Association, 19(1), 33-46.

Huang H, Gartner G, Schmidt M, Li Y (2009) Smart Environment for Ubiquitous Indoor Navigation. International Conference on New Trends in Information and Service Science, NISS '09: $176-180$ 
Jensen CS, Lu H, Yang B (2009) Graph Model Based Indoor Tracking. Proceedings of the 2009 Tenth International Conference on Mobile Data Management: Systems, Services and Middleware: 122-131

Karas I, Batuk F, Akay A, Baz I (2006) Automatically Extracting 3D Models and Network Analysis for Indoors. In Abdul-Rahman A., Zlatanova S., Coors V (Ed.): Innovation in 3DGeo Information System. Springer, Berlin Heidelberg: 395-404

Lee J (2001) 3D Data Model for Representing Topological Relations in Urban Features. Delaware County Regional Planning Commission, Delaware. http://gis.esri.com/library/userconf/proco1/professional/papers/pap565/p565.htm. Accessed 13 May 2011

Lee $\mathrm{J}$ (2004) A spatial access-oriented implementation of a 3-D GIS topological data model for urban entities. GeoInformatica 8 (3): 237-264

Lee, J. (2009). GIS-based geocoding methods for area-based addresses and 3 D addresses in urban areas. Environment and planning B, Planning and design, 36(1), 86-106.

Li Y, He Z (2008) 3D Indoor Navigation: a Framework of Combining BIM with 3D GIS. Proceedings of the 44th ISOCARP Congress 2008

Löchtefeld, M., Gehring, S., Schöning, J., \& Krüger, A. (2010). PINwI: pedestrian indoor navigation without infrastructure. Proceedings of the 6th Nordic Conference on HumanComputer Interaction: Extending Boundaries.

Lyardet F, Szeto DW, Aitenbichler E (2008) Context-Aware Indoor Navigation. In: Hutchison D, Kanade T, Kittler J, Kleinberg JM, Kobsa A, Mattern F, Mitchell JC, Naor M, Nierstrasz O, Rangan CP, Steffen B, Sudan M, Terzopoulos D, Tygar D, Weikum G (Ed.) Ambient Intelligence. Springer, Berlin Heidelberg 5355/2008:290-307

Mautz R, Kunz M, Ingensand H (2010) Proceedings of the 2010 International Conference on Indoor Positioning and Indoor Navigation (IPIN). IEEE Xplore: CFPIoogJ-ART. doi: 10.1109/IPIN.2010.5648185

Meijers M, Zlatanova S, Pfeifer N (2005) 3D Geo-Information Indoors: Structuring for Evacuation. Proceedings of Next generation 3D City Models.

Nagel, C., Becker, T., Kaden, R., Li, K.-J., Lee, J., \& Kolbe, T. H. (2010). Requirements and Space-Event Modeling for Indoor Navigation, OGC Discussion Paper (pp. 54). OGC: OGC.

Pfaff P, Kümmerle R, Joho D, Stachniss C, Triebel R, Burgard W (2008) Navigation in Combined Outdoor and Indoor Environments using Multi-Level Surface Maps. Department of Computer Science, University of Freiburg. http://citeseerx.ist.psu.edu/viewdoc/summary?doi=10.1.1.69.9146 Accessed 13 May 2011

Ran L, Helal S, Moore S (2004) Drishti: An Integrated Indoor/Outdoor Blind Navigation System and Service. Second IEEE International Conference on Pervasive Computing and Communications (PerCom'04) 
Retscher G, Thienelt M (2004) NAVIO - A Navigation and Guidance Service for Pedestrians. Journal of Global Positioning Systems 3(1-2): 208-217

Scooter W, Helal S (2005) RFID Information Grid for Blind Navigation and Wayfinding. Proceedings of the Ninth IEEE International Symposium on Wearable Computers: 34-37

Slingsby A, Raper J (2007) Navigable Space in 3D City Models for Pedestrian. In van Oosterom P, Zlatanova S, Penninga F, Fendel E (Ed.) Advances in 3D Geoinformation Systems. Springer, Berlin Heidelberg: 49-64

Tsetsos V, Anagnostopoulos C, Hadjiefthymiades S (2007) Semantic Enrichment of Location-Based Services. In Taniar D (Ed.) Encyclopedia of Mobile Computing and Commerce: 856-862

Walton, L. A., \& Worboys, M. (2009). Indoor Spatial Theory. 6 pp. Retrieved from www.spatial.maine.edu/ISAmodel/documents/IST_ISAo9.pdf

http://blumenthals.com/blog/2009/10/12/google-replaces-tele-atlas-data-in-us-withgoogle-data/

http://ifgi.uni-muenster.de/archives/photomap/Home.html

www.makkamappa.com 